\title{
EXPERIMENTS WITH A MESOMASER
}

\author{
W. LANGe aNd II. WaLthIER \\ Max-Planck-Institut für Quantenoptik \\ IIans Kopfermann-Str. 1, 85748 Garching bei München, Germany
}

\begin{abstract}
We investigated the dynamically modified interaction between Rydberg a ioms and a single mode of a microwave cavity driven by a strong external field. With a monochromatic injected field we observed dynamic suppression of spontaneous emission, when the intracavity Rabi frequency exceeds the cavity linewidth. We discuss a theoretical model using non-Markovian decay rates, which is in excellent agreement with experiment. When a bichromatic driving field is used, enhanced decay reappcars in narrow resonance zones as the detunings and injected intensities are varied. These results are interpreted in terms of cavity-modified transitions between the Floquet states of a two-level atom. Striking aspects of these transitions are their small linewidth and an asymmetry between emission and absorption.
\end{abstract}

PACS numbers: $42.50 . \mathrm{IIz}, 32.70 . \mathrm{Jz}, 32.80 .-\mathrm{t}$

\section{Introduction}

The interaction of a two-level atom with a single mode of the electromagnetic field in a cavity is the most fundamental system for investigating the coupling between matter and radiation. It was first treated theoretically by Jaynes and Cummings [1] and has received a lot of attention in recent years, as a large number of experiments related to this model have been accomplished. Three rates govern the dynamics of the system: the atom-field coupling constant $g$, which sets the time-scale of the coherent evolution, the damping rate of the cavity field $\kappa$ and, in the absence of atomic relaxation, the inverse interaction time $T^{-1}$. When the coupling $g$ is much stronger than the cavity damping $\kappa$, the Jaynes-Cummings model predicts an oscillatory energy exchange between the atom and the cavity mode. An important system suitable to explore this strong coupling regime is the one-atom maser [2,3]. Here, the dynamics of the photon exchange between an atom and a single mode of a microwave cavity can be investigated with less than one atom in the cavity at a time. When a large atomic flux is used, a steady state field builds up in the cavity which can exhibit nonclassical properties, like sub-Poissonian photon statistics [4]. An essential requirement for the generation of such a steady state field is that many atoms pass through the cavity within the cavity decay time.

In the optical region, the signature of the photon exchange is a splitting of the normal modes of the atom-field system, which has been observed with sodium 
atoms in a Fabry-Perot resonator $[5,6]$. Recently, the phenomenon has also been demonstrated in the microwave regime [7].

When, on the other hand, the case of a microwave cavity with a decay rate larger than the atom-field coupling is considered, the system dynamics is entirely different from the michomaser. A photon emitted into the cavity mode is dissipated before it can be reabsorbed by the atom or interact with another atom. Therefore, the atoms cannot build up an electromagnetic field inside the cavity like in the strong coupling case. For weak coupling, the influence of the cavity on the atom can be described by perturbation theory. Such a system is suited to investigate cavity-induced modification of radiative atomic decay.

It was first realized by Purcell [8] that the spontaneous emission rate is not only determined by the coupling between atom and field, but also by the mode density of the electromagnetic environment. In a cavity resonant with the atomic transition, the density of modes at the transition frequency is much higher than in free space. Consequently, spontaneous emission is strongly enhanced by the cavity. On the other hand, when the cavity is detuned from the atomic resonance, decay is inhibited. Since there is no mode available for the atom to interact with, it cannot emit the stored energy and the lifetime in the excited state is increased. Both enhancement and inhibition of spontaneous emission have been observed in the optical domain [9] as well as with microwave transitions between highly excited Rydberg levels [10-12].

In the one-atom maser and other microwave cavity-QED experiments reported so far, the cavity or cavity-like structure initially only contains the vacuum field or in some cases a few thermal photons. The cavity is pumped exclusively by atoms injected in the upper state. In this paper we discuss experiments, in which the cavity mode is excited by an external driving field [13]. This introduces intensity and detuning of the driving field as new system parameters and leads to novel effects. We consider a driven atom-cavity system in the special case of cavity decay rate $\kappa$ and coupling constant $g$ having the same order of magnitude, i.e. the case in between the strong and the weak coupling limit. This allows us to investigate nonlinear effects at small photon numbers, but still use a theoretical model based on the perturbative approach.

The observation of coherent and nonlinear phenomena in this regime requires the presence of more than one atom $(N>1)$ in the cavity at a time. Moreover, as photons are supplied by an external source, the occupation number $n$ of the cavity mode can become quite large. Such a system may be called mesoscopic, because it represents an intermediate regime between the microscopic $(N \leq 1)$ and the macroscopic $(N \gg 1)$ description.

Two limiting cases are of particular interest: that of a weak and that of a strong external driving field. If the external excitation of the cavity mode is smaller than or comparable to the field radiated by the atoms, cooperative atomic behavior is expected. Among the predictions for this regime are multistable steady states, self-pulsing [14], and phase bimodality of the atom-cavity system [15, 16]. 
In this article we will focus on the strong field case, where the external driving is so strong that the Rabi frequency associated with the cavity field exceeds the cavity linewidth

$$
\Omega=2 g \sqrt{n} \gg \kappa,
$$

$n$ being the number of photons in the cavity mode and $g$ is the atom-field coupling. Clearly, in this case, the atomic contribution to the cavity field is negligible. The power in the mode is determined exclusively by the intensity of the external field and a Lorentzian factor taking into account the detuning of the external field with respect to the cavity frequency. In this situation cooperative effects like multistability cannot arise.

Our analysis shows that the dynamics in the mesoscopic strong field regime is well described in terms of cavity-modified decay rates. We have investigated theoretically and experimentally the behavior of these rates for intense driving $[13,17]$.

An important result is that decay depends on the strength of the external field in a way which at first sight is surprising: the larger the driving field, the smaller is the cavity-emhanced spontaneous emission rate. We have found that about 100 photons in the cavity are sufficient to completely suppress spontaneous emission as well as thermally induced transitions.

Such a dynamic modification of spontaneous emission has been predicted for the optical domain some time ago by Lewenstein, Mossberg, and Glauber [18], yet the experiment they have suggested has not been realized. The main difficulty is that only emission into the cavity mode is dynamically suppressed, while atoms can still decay into the continuum of side modes not affected by the cavity. Since an ordinary optical cavity only encloses a small solid angle, cavity related effects are entirely masked by decay into side modes. This problem does not occur with the microwave cavities used in the Rydberg atom experiments, as these are completely closed, so that atoms exclusively interact with a single cavity mode. It should be noted that for a closed cavity, the external driving field necessarily is injected into the cavity mode. In contrast, in corresponding optical experiments the laser usually occupies a side mode not supported by the cavity.

There is a simple way of understanding the suppression of spontaneous emission by a monochromatic driving field. The system can be described in terms of semiclassical dressed states [19-21]. In free space and for resonant driving one obtains the Mollow resonance fluorescence triplet [22] depicted in Fig. 1. This has to be compared with the spectral density of the cavity mode, represented by the dashed line in Fig. 1. In the strong driving situation considered here, the Rabi frequency $\Omega$ associaled with the external field exceeds the cavity linewidth $\kappa$ (cf. Eq. (1)). Consequently, the fluorescence side bands fall outside the response spectrum of the cavity mode, which results in a strong suppression of the cavity-induced decay rate. Transitions corresponding to the central peak of the fluorescence triplet do not change the atomic states detected in Rydberg experiments and hence do not contribute to the decay of atomic population. If the field is sufficiently strong, complete decoupling of the atom from the cavity mode can be achieved. 


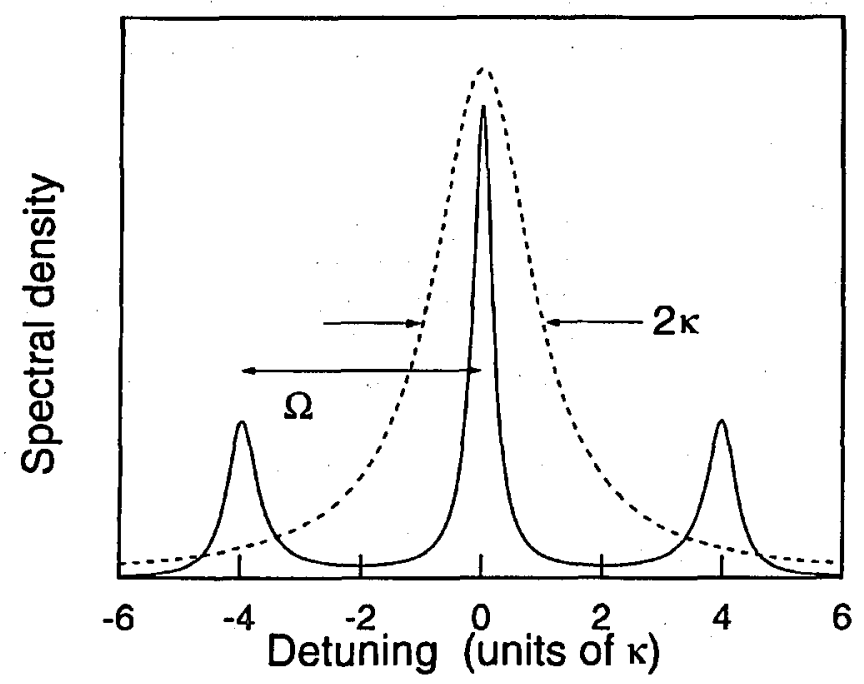

Fig. 1. Mollow triplet of fluorescence in free space (solid curve). In a driven cavity, fluorescence is dynamically modified when the side bands fall outside the cavity resonance line (dashed curve).

This result can be put on a quantitative basis by calculating the renormalization of the cavity-induced spontaneous emission rates by the strong field [17]. It is important to note that the cavity mode into which the atom emits has a finite response time $\kappa$. Therefore, atomic decay induced by the cavity may be considered as a non-Markovian process, depending on the dynamics of the atomic variables during a cavity damping time $r$. As the time scale for the evolution of the driven system is given by the Rabi frequency $\Omega$, modified decay rates are expected for $\Omega>\kappa$, in agreement with the simple argument above.

The physical situation becomes considerably more complex when the single frequency driving field is replaced by a bichromatic field. Recent experimental [23] and theoretical [24-26] investigations have shown that the free space fluorescence spectrum of a two-levcl atom in a strong bichromatic field fundamentally differs from the spectrum observed in the monochromatic case. An example is shown in Fig. 2. Instead of the three peaks characteristic of monochromatic fluorescence (Fig. 1), there now is a large number of side bands. Their most striking property is that the peak separation is no longer dependent on the Rabi frequency, but is equal to half the difference between the two driving frequencies. The Rabi frequency here only determines the amplitude envelope of the resonances.

The complex structure of these spectra is a consequence of the fact that the IIamiltonian of a system with bichromatic driving is necessarily time dependent. Therefore, stationary energy levels and steady-state spectra do not exist and all expectation depend on time. From these free space results we expect that cavity-induced decay under bichromatic driving shows features not present in the single frequency case. 


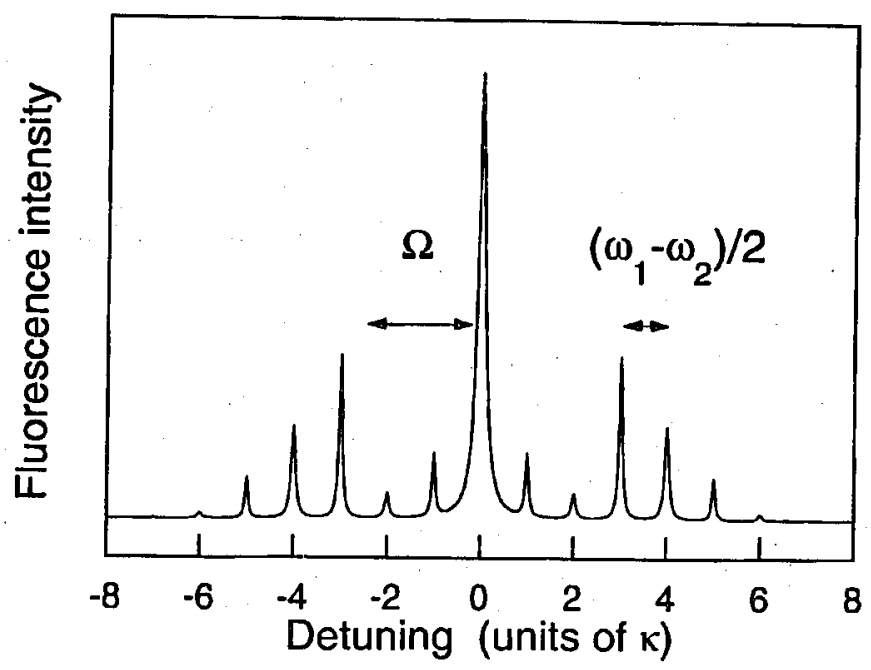

Fig. 2. Theoretical free space fluorescence spectrum of a two-level atom in a bichromatic field [24]. The fields are assumed to have equal Rabi frequencies.

The spectra we have obtained by driving the cavity with two frequencies, indeed, differ from the monochromatic results. Although for two frequencies suppressed decay also prevails, an additional feature appears in the spectra: at certain detunings of the driving fields resonances in the decay rate occur. Their position depends on the intensities of both driving ficlds and the cavity frequency. The width of the resonant structurcs can bccome extremely small and even drop below all characteristic widths of the system, like the cavity linewidth, transit broadening, and inhomogeneous broadening. In addition, there is a strong asymmetry between upper and lower level decay.

We have obtained a theoretical interpretation of these experimental results, describing the atom-field system in terms of Floquet states [27]. By this method we transform the two-level system wilh time dependent fields to an infinite number of stationary quasi-energy levels, interacting with the cavity mode. We show that the data we present can be regarded as the first observation of cavity-modified transitions among Floquet states of an atom.

In Sec. 2 we give details of the new Rydberg atom experiment investigating the mesoscopic regime. The experimental data for monochromatic driving are presented in Sec. 3, demonstrating the dynamic modification of atomic emission characteristics. In this section we also give a theoretical interpretation of our results, based on a semiclassical dressed state analysis of the system. The novel effects obtained under strong bichromatic driving are discussed in Sec. 4. In Sec. 5 we outline the theoretical treatment of the bichromatic case in terms of Floquet states. Finally, in Sec. 6 we describe the observed asymmetry between emission and absorption in the system. 


\section{Expcriment}

The experimental apparatus used to investigate the mesoscopic regime of cavity-QED is similar to the one-atom maser [4]. We employ a microwave transition between neighboring levels of ${ }^{85} \mathrm{Rb}$ Rydberg atoms, excited to a state of high principal quantum number (here $n=53$ ). Riydberg states are well suited for investigating the interaction of matter and radiation. First, we can take advantage of the large atom-field coupling of transitions between Rydberg levels scaling as $n^{2}$. Second, transition frequencies are in the microwave region. Therefore, we can use RF cavities, operated in a fundamental mode. Unlike the Fabry-Perot resonators employed in optical experiments, our microwave cavity encloses the full solid angle. This way, coupling to side modes is eliminated and the interaction is restricted to the one cavity mode resonant with the atomic transition. As only two Rydberg states are coupled resonantly by the cavity field, the Rydberg atoms can in good approximation be considered as two-level atoms. Another advantage of the microwave regime is the long interaction time of the atoms in the cavity.

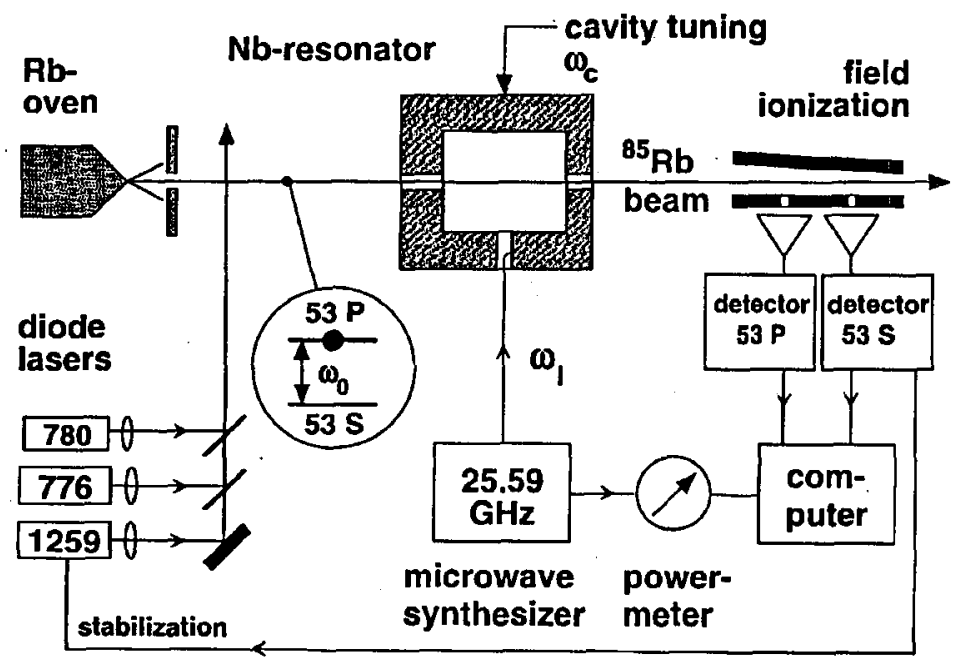

Fig. 3. Scheme of the experimental setup for the case of monochromatic external driving (frequency $\omega_{l}$ ) of the cavity. The wavelengths (in $\mathrm{nm}$ ) of the diode lasers used for the excitation of the 53P Rydberg state are indicated.

Our experimental setup is shown in Fig. 3. A collimated beam of rubidium atoms is excited to the $53^{2} P_{3 / 2}$ state of ${ }^{85} \mathrm{Rb}$ by means of three-step diode laser excitation with wavelengths $780.2 \mathrm{~nm}, 776.0 \mathrm{~nm}$ and $1258.8 \mathrm{~nm}$, respectively. The atomic beam has a thermal velocity distribution, the most probable time of flight through the cavity being $\tau=67 \mu \mathrm{s}$. Due to the long lifetime of the Rydberg levels, the linewidth of the atomic transition is determined by transit time broadening.

After being excited to the $53^{2} P_{3 / 2}$ state, the atoms traverse a superconducting cylindrical cavity along its axis. The $\mathrm{TM}_{020}$-mode of the cavity is in resonance 
with the $53^{2} P_{3 / 2} \rightarrow 53^{2} S_{1 / 2}$ transition at a frequency $\omega_{0}=2 \pi \times 25.5903 \mathrm{GHz}$. The atom-field coupling constant between the $m= \pm \frac{1}{2}$ magnetic substates was calculated to be $g=2 \pi \times 17 \mathrm{kHz}$.

The cavity is made of superconducting niobium. By cooling to a temperature of $4.2 \mathrm{~K}$ in a ${ }^{4} \mathrm{He}$-cryostat, a loaded quality factor $Q$ of $1.9 \times 10^{6}$ was obtained. This corresponds to a cavity decay rate $\kappa=2 \pi \times 6.7 \mathrm{kHz}$. This relatively large decay rate distinguishes our experiment from the one-atom maser, where $\kappa$ is much smaller than the coupling constant $g$.

A further distinction from the one-atom maser is related to the cavity mode employed. While the maser cavity is operated in the $\mathrm{TE}_{121}$-mode with a sinusoidal variation of the electric field along the axis, for the present experiments the $\mathrm{TM}_{020}$-mode was chosen. It has the advantage of providing a constant amplitude of the electric field along the axis of the cavity. In this way we avoid effects relating to position dependent atom-field coupling. At the entrance and exit holes, power leakage leads to a gradual turn on and turn off of the field amplitude in the rest frame of the atoms. The corresponding rise time is about $5 \mu \mathrm{s}$. The cavity field is linearly polarized with the electric field vector parallel to the direction of the atomic beam.

The one-atom maser cavity is pumped only by atoms injected in the upper state. As we are interested in the dynamics of a strongly driven system, our cavity field is supplied by an external microwave source. Coupling is achieved through a hole in the sidewall, equidistant from the two end caps. We did not use the atomic beam holes for feeding the microwaves, to avoid premature interaction of atoms and field. The microwave source is a $26 \mathrm{GHz}$ synthesizer with a linewidth below $1 \mathrm{kHz}$. For the experiments with a bichromatic driving field, we used an additional synthesizer at $13 \mathrm{GIIz}$ in conjunction with a frequency doubler. IIere, the linewidth achieved was about $2 \mathrm{IIz}$. We did not lock the phases of the two synthesizers, as their relative stability was larger than the inverse transit time of the atoms.

Owing to the closed cavity configuration, the observation of atomic fluorescence in the cavity is ruled out. The only source of information available on the atom-cavity interaction is the state of the atoms leaving the cavity. The Rydberg atoms are detected by state-selective field ionization, using two secondary electron multipliers with an efficiency of about $10 \%$ [4].

\section{Dynamic suppression of spontaneous emission by a strong driving field}

In the mesomaser experiments the cavity decay rate $\kappa$ is about equal to the coupling constant $g$. Therefore, oscillatory exchange of energy between atom and cavity mode does not occur, and it is appropriate to describe the influence of the cavity in terms of modified atomic decay rates, which can be obtained from perturbation theory.

When no external microwave field is applied, we expect enhanced or inhibited spontaneous emission [10], depending on the atom-cavity detuning. In the microwave regime, transitions induced by blackbody radiation also contribute, because of the large mean number $\bar{n}=2.9$ of thermal photons in the cavity mode 
at a temperature of $4.2 \mathrm{~K}$. The blackbody induced transition rate simply adds to the spontaneous rate. Thus the enhanced decay rate is given by [17]:

$$
\Gamma_{\|}=\frac{2 g^{2} \kappa}{\kappa^{2}+\left(\omega_{\mathrm{c}}-\omega_{0}\right)^{2}}(1+2 \bar{n}) \text {. }
$$

Since for $\omega_{\mathrm{c}}=\omega_{0}$, the inverse decay rate $\Gamma_{\|}^{-1}$ is small compared with the interaction time $T$, the atoms leave the cavity in thermal equilibrium when the cavity is tuned into resonance with the atomic transition. This is demonstrated by the field ionization spectrum in Fig. 4a. There are peaks in the Rydberg count rate at two

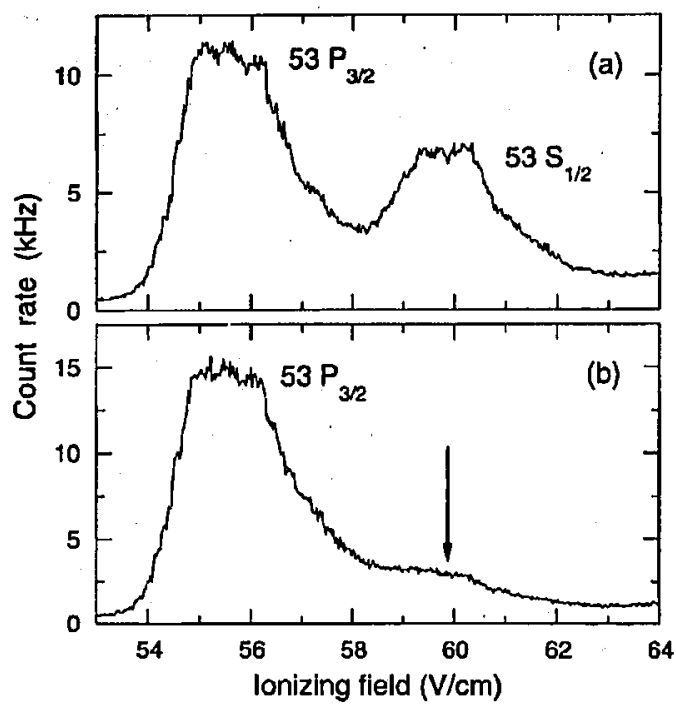

Fig. 4. Field ionization spectra of atoms prepared in state $53^{2} P_{3 / 2}$, passing through a cavity resonant with the transition to $53^{2} S_{1 / 2}$. Atoms leaving the cavity in the $53 P$ $(53 S)$ state are ionized in a field of $56(60) \mathrm{V} / \mathrm{cm}$. (a) Without external driving. The population of level $53 S$ indicates spontaneous and thermally induced transitions. (b) With strong external driving. The $53 S$ signal (arrow) has alınost completely disappeared, owing to the dynamic suppression of spontaneous and thermally induced transitions.

values of the ionizing field, corresponding to atoms in the initially occupied $53 P$ level and the lower $53 S$ level, respectively. The lower level count rate is a measure for cavity-induced transitions.

To investigate the new regime of a driven cavity, we injected a quasi-resonant external microwave field into the cavity mode. This is in contrast to optical experiments, where the driving field usually is injected in a side mode not supported by the cavity. We monitored the lower level count rate as a function of the microwave intensity. The result is shown in Fig. 5. At $-100 \mathrm{dBm}$ we still observed the full enhanced decay rate. At a power level of $-73 \mathrm{dBm}$, corresponding to only about 100 microwave photons in the cavity, we registered a sharp drop in the number of atoms leaving the cavity in the lower level. The fact that a relatively 


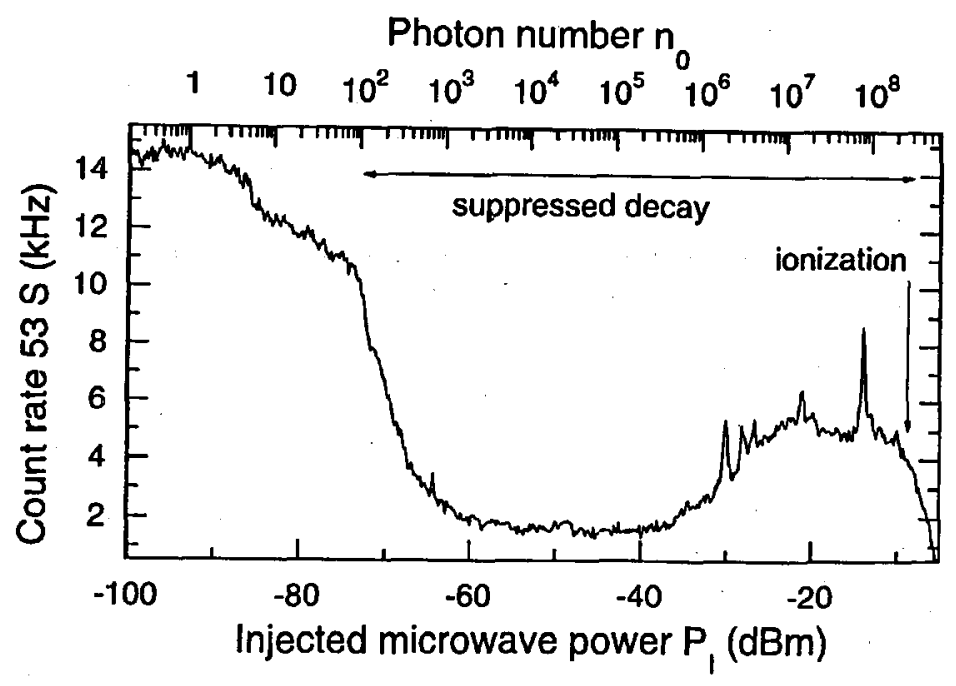

Fig. 5. Count rate of atoms in the lower Rydberg level (53S) as a function of the intensity of the external inicrowave field close to resonance. The corresponding number $n_{0}$ of photons occupying the cavity mode is displayed as the top axis.

small number of photons can lead to drastic changes in the system behavior is a consequence of the strong coupling of Rydberg atoms to the electromagnetic field. At still higher input intensities of up to $10^{6}$ photons in the cavity, the number of lower level atoms dropped quickly to a value close to zero. The field ionization spectrum obtained in this case is displayed in Fig. $4 \mathrm{~b}$. The peak corresponding to lower Rydberg-level (53S) atoms, has almost completely vanished, even though the intensity of the resonant cavity field far exceeds the saturation threshold of the $53 P \rightarrow 53 S$ transition. This is the first observation of suppression of atomic decay due to the system dynamics induced by a strong driving field, as predicted in Ref. [18]. At still higher intensities, the lower level count rate rises again and displays resonances, indicating the breakdown of the two-level approximation.

Qualitatively, the observed suppression of decay can be understood in the dressed atom picture. If the Rabi frequency of the driving field is larger than the cavity linewidth, the side bands of the fluorescence triplet are shifted outside the cavity response spectrum (cf. Fig. 1) and hence atomic decay is suppressed. The central component at the driving field frequency corresponds to transitions which do not change the atomic population. Therefore, they are not detected with our sctup, which is only sensitive to clianges in the occupation of atomic levels.

A rigorous theoretical analysis of the experiment was reported in a recent paper [17]. It starts from the IIamiltonian of a two-level atom of frequency $\omega_{0}$ coupled to a single mode cavily field of frequency $\omega_{c}$, i.e. the Jaynes-Cummings IIamiltonian. In addition, the cavity is driven by an external field of frequency $\omega_{l}$ and amplitude $E$. In a frame rotating at the frequency $\omega_{l}$ the Hamiltonian is

$$
I I=\hbar \Delta S^{z}+\hbar \delta a^{\dagger} a+\hbar g\left(S^{+} a+S^{-} a^{\dagger}\right)+\hbar\left(a E^{*}+a^{\dagger} E\right),
$$


where the detunings $\Delta$ and $\delta$ are defined by

$$
\Delta=\omega_{0}-\omega_{l}, \quad \delta=\omega_{c}-\omega_{l} .
$$

The annihilation and creation operators $a$ and $a^{\dagger}$ are associated with the cavity field, while $S^{ \pm}$and $S^{z}$ are the spin- $\frac{1}{2}$ angular momentum operators describing the two-level atom.

Cavity relaxation at the finite temperature $T$ is handled by the master equation for the density matrix $\rho$ of the combined atom-field system

$$
\begin{aligned}
& \frac{\mathrm{d} \rho}{\mathrm{d} t}=-\frac{\mathrm{i}}{\hbar}[H, \rho]+\mathcal{L} \rho, \\
& \mathcal{L} \rho=-\kappa(1+\bar{n})\left(a^{\dagger} a \rho-2 a \rho a^{\dagger}+\rho a^{\dagger} a\right)-\kappa \bar{n}\left(a a^{\dagger} \rho-2 a^{\dagger} \rho a+\rho a a^{\dagger}\right) .
\end{aligned}
$$

As shown above, a typical value for the mean photon number in the vavity mode is $10^{5}$. At those intensities, a classical treatment of the coherent excitation of the cavity field is justified. On the other hand, the quantum description of the cavity mode is still needed to take into account spontaneous emission and the thermal photons in the cavity. Therefore, it is advantageous to separ we the coherent and the incoherent part of the cavity field. This can be done by a unitary transformation, leading to a description in which the atom is coupled directly to the classical external field, while the quantized cavity field is in the state of thermal equilibrium $[17,28]$. The transformed Hamiltonian reads

$$
H^{\prime}=\hbar \Delta S^{z}+\hbar \delta a^{\dagger} a+\hbar g\left(S^{+} a+S^{-} a^{\dagger}\right)+\frac{\hbar}{2}\left(S^{+} \Omega+S^{-} \Omega^{*}\right) .
$$

The quantity $\Omega$ is the Rabi frequency of the classical driving field, which is given by

$$
\Omega=\left|\frac{2 g E}{\kappa+\mathrm{i} \delta}\right| .
$$

The Lorentzian denominator appearing in (7) reflects the fact that the external field $E$ is injected directly into the cavity mode and hence couples less strongly for large field-cavity detuning.

The classical strong driving field can be treated most elegantly by working in the basis of semiclassical dressed states $| \pm\rangle[19-21,29]$. These states are defined in terms of the bare atomic states $|1\rangle$ and $|2\rangle$ by the equation

$$
\begin{aligned}
& |+\rangle=\cos \theta|1\rangle+\sin \theta|2\rangle, \\
& |-\rangle=-\sin \theta|1\rangle+\cos \theta|2\rangle,
\end{aligned}
$$

where the Stueckelberg angle $\theta$ is defined by $\tan (2 \theta)=\Omega / \Delta$.

Decay rates for the dressed levels are calculated using a second order Born approximation with respect to the coupling constant $g$ to simplify master equation (5). Subsequently, the cavity field is eliminated adiabatically by tracing over the variables $a$ and $a^{\dagger}$. From the resulting master equation for the atom, one can derive equations of motion for the Bloch vector $\boldsymbol{R}$ in the dressed state basis, which assume a particularly simple form in the rotating wave approximation

$$
\left\langle\dot{R}^{+}\right\rangle=\left(\mathrm{i} \tilde{\Omega}-\Gamma_{\perp}\right)\left\langle R^{+}\right\rangle
$$




$$
\left\langle\dot{R}^{z}\right\rangle=-\Gamma_{\|}\left(\left\langle R^{z}\right\rangle-\left\langle R_{\mathrm{ss}}^{z}\right\rangle\right) .
$$

The quantity $\tilde{\Omega}$ is the dynamically modified Rabi frequency, $\Gamma_{\perp}$ and $\Gamma_{\|}$are decay rates. The dressed state inversion relaxes to its steady state value $\left\langle R_{\mathrm{ss}}^{z}\right\rangle$. The parameter relevant for the interpretation of the experimental results is $\Gamma_{\|}$, the rate at which the dressed state population inversion $\left\langle R^{z}\right\rangle$ decays

$$
\Gamma_{\|}=2 g^{2}(1+2 \bar{n})\left[\frac{\kappa \cos ^{4} \theta}{\kappa^{2}+(\delta-\bar{\Omega})^{2}}+\frac{\kappa \sin ^{4} \theta}{\kappa^{2}+(\delta+\bar{\Omega})^{2}}\right],
$$

where $\bar{\Omega}=\sqrt{\Delta^{2}+\Omega^{2}}$ is the generalized Rabi frequency. As expected, for a resonant cavity and a resonant driving field $(\Delta=\delta=0)$, one finds that the decay of the dressed state population is strongly suppressed compared to the zero field result by a factor $(\Omega / \kappa)^{2}$. Therefore, if the atom is in a dressed state, the spontaneous emission rate can be made arbitrarily small by increasing the Rabi frequency $\Omega$ of the driving field.

An intriguing aspect of the problem is that intensity dependent decay rates may be regarded as originating from a non-Markovian process $[18,17]$. The finite response time of the cavity mode leads to decay terms in the modified Bloch equations, which depend on atomic variables at earlier times. The decay rate $\Gamma_{||}$is proportional to a sum containing Laplace transforms of atomic correlation functions, for example

$$
\int_{0}^{\infty} \mathrm{e}^{-(\kappa-\mathrm{i} \delta) \imath}\left\langle S^{+}(t-\tau) S^{-}(l)\right\rangle \mathrm{d} \tau .
$$

This expression clearly violates the Markov assumption, as it implies a reservoir memory over the cavity response time. The decay rates become depender.t on the intensity of the driving field, because the dynamics of the Bloch vector $S(l)$ appearing in (12) is governed by the Rabi oscillations. If the Rabi frequency exceeds the cavity linewidth $\kappa$, averaging of the correlation function $\left\langle S^{+}(t-\tau) S^{-}(t)\right\rangle$ over a time $\kappa^{-1}$ reduces expression (12), and hence the decay rate $\Gamma_{\|}$, to almost rèro. This illustrates how non-Markovian dynamics can lead to the suppression of decay in a strongly driven system interacting with a frequency dependent reservoir.

An important question concerns transient effects in the atom-field interaction during the entrance and exit phase. Atoms are prepared outside the cavity in the upper bare state. When an atom enters the cavity field, this state is mapped to a superposition of dressed levels. The reverse process occurs at the cavity exit, as atoms are detected after they have left the cavity. To examine transient behavior, the Bloch equations (3) were integrated for a time dependent coupling constant $g(t)$. This way the turn on and turn off of the coupling in the rest frame of the atoms during their flight through the cavity were modeled.

The results of the calculations show that the dynamics induced by the time dependent coupling critically depends on the detuning of the driving field with respect to atoms and cavity. One can distinguish between a diabatic and an adiabatic regime.

If the detuning between atom and driving field is larger than the inverse rise time of the cavity field, the atoms enter the cavity adiabatically. Starting in the upper level $(53 P)$ at zero field, each atom slowly passes through a succession 
of stationary states on the way into the cavity. Consequently, inside the cavity the atom is in one of the dressed states $| \pm\rangle$. By analogy, an atom leaving the cavity in the same dressed state ends up in the initially occupied $53 P$ level. This explains the surprising fact that the strong resonant field itself does not induce any transitions. Atoms detected in the lower $(53 S)$ level are exclusively due to the decay of the dressed state population inside the cavity and hence are governed by the rate $\Gamma_{\|}$(Eq. (11)). This is in perfect agreement with the experimental result that atomic decay is suppressed if the driving field is strong enough.

If the detuning between atom and driving field is less than the inverse rise time of the cavity field, the splitting between the semiclassical dressed states is so small that the change of this splitting on entry (or exit) is fast enough for Landau-Zener type transitions between dressed states to occur. This contribution to the change of atomic population is independent of cavity-induced effects. We measured the lower level population at the cavity exit as a function of the detuning of the driving field (Fig. 6a). The diabatic contribution to the overall transition

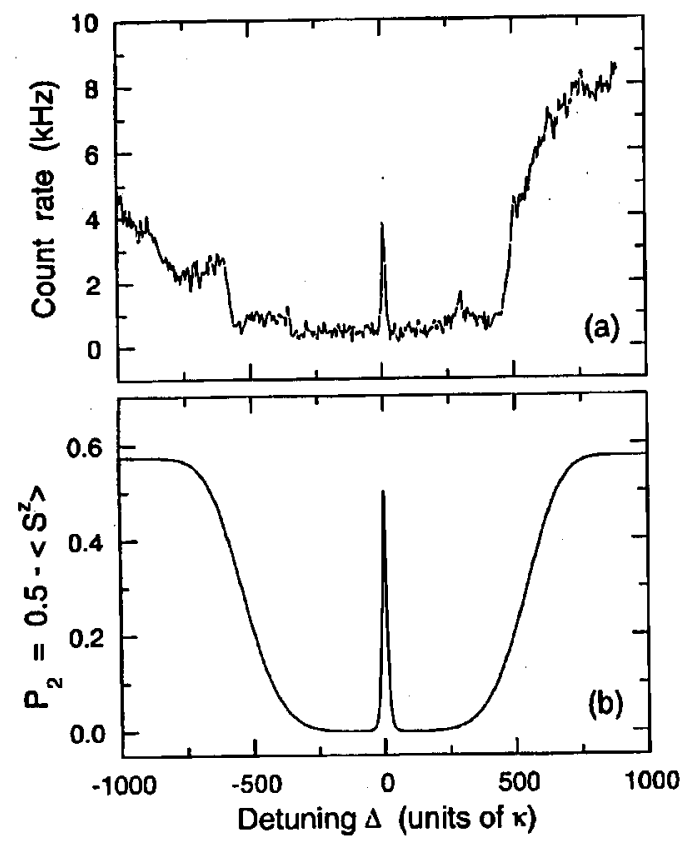

Fig. 6. (a) Experimental trace of the lower level population for a driving field amplitude $E=15000 \kappa$. The atomic beam has a thermal velocity distribution with an average interaction time $T=2.6 \kappa^{-1}$. (b) Corresponding theoretical result, obtained by integrating the modified Bloch equations (9-10).

rate appears as a peak at the atomic frequency with a width of $10 \kappa$, which is roughly the inverse rise time. The spectrum is in excellent agreement with the result from the theoretical model (Fig. 6b). 
A striking fcature in Fig. 6 is that for large detuning the suppression of spontaneous emission vanishes. This is a simple consequence of Eq. (7) for the intracavity Rabi frequency. As the external field is injected into the cavity mode, the excitation level of the mode decreases with the detuning of the driving field according to a Lorentzian. In optical experiments, where the driving field is injected into a side mode, this restriction is absent. From Eq. (11) follows a critical detuning:

$$
\Delta_{\mathrm{c}}=\sqrt[3]{\frac{2 g^{2} E^{2}}{\kappa}}
$$

at which the decay rate has dropped to half its zero-field value. We have tested experimentally the dependence of the critical detuning $\Delta_{c}$ or, equivalently, the width $w$ of the dynamic suppression region, on injected power (Fig. 7). We found good agreement with the theoretical slope for strong fields above $-40 \mathrm{dBm}$.

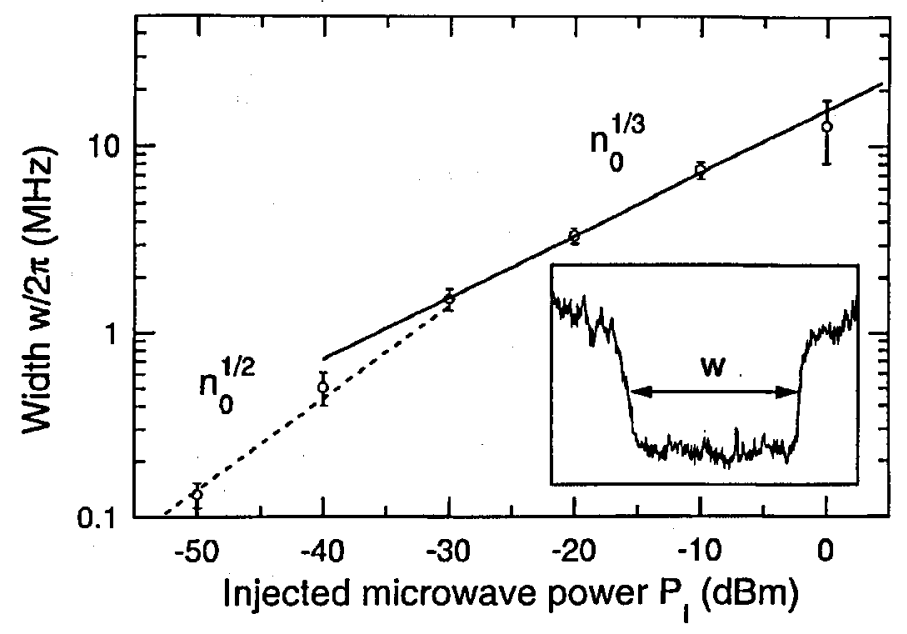

Fig. 7. Measured width of the range where dynamic suppression is observed. The solid line represents the theoretical result in the stron: field case $(\Omega \gg \kappa)$.

\section{Cavity-induced transitions in a strong bichromatic field}

The modification of cavity-induced transitions for a single frequency driving field is limited to the suppression of decay for large intensities. This is due to the simple structure of the dressed states in a monochromatic field. A much richer dynamics can be expected when we employ a field containing two frequency components.

We have probed cavity-induced effects under the influence of strong bichromatic driving by performing an experiment analogous to the monochromatic case, but with an additional microwave field injected into the cavity. Intensity and frequency of the second field were controlled independently from the first field. This is in contrast to most experiments on bichromatic driving in the optical domain, 
where both fields are symmetrically detuned from the atomic resonance and assumed to have equal intensity.

Like in the monochromatic experiments we injected the atoms in the upper level and took spectra of the lower level count rate at the cavity exit. It is important for the interpretation of our results to clarify the procedure for obtaining these spectra. For practical reasons we scanned the frequency of only one field (called $\omega_{1}$ ), while keeping the frequency of the second field $\left(\omega_{2}\right)$ fixed. At the same time the injected intensities of both external ficlds were kept constant. Note, however, that there is an inherent variation of the intracavity amplitude of the scanned field, as it depends on the mode density and according to Eq. (7) varies like the Lorentzian cavity line shape.

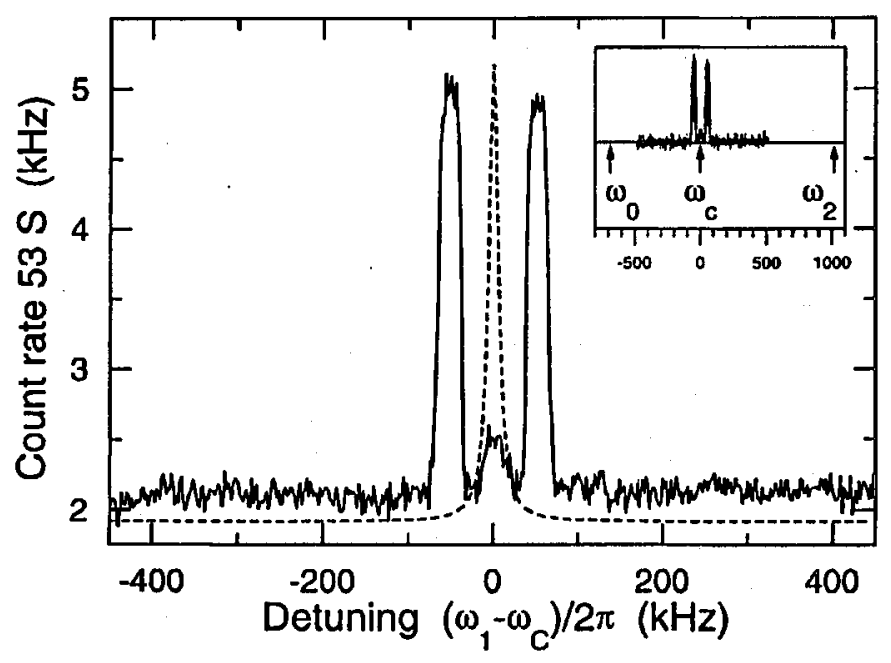

Fig. 8. Count rate of atoms in the lower Rydberg level (53S) for a bichromatic driving field. The inset shows the detuning of the second field $(1018 \mathrm{kIz})$ and the atomic transition frequency $(-681 \mathrm{kIIz})$ with respect to the cavity resonance. The intracavity Rabi frequency of the first field at the cavity resonance $\left(\omega_{1}=\omega_{c}\right)$ is $120 \mathrm{MHz}$, that of the second field at $\omega_{2}$ is $15 \mathrm{MHz}$.

A typical spectrum obtained in this way is presented in Fig. 8. The baseline of the lower level count rate, which is close to zero, is the signature for suppressed atomic decay as it was observed for monochromatic driving. There is, however, a striking feature not present in the single frequency spectra. A pronounced pair of narrow resonances in the number of lower level atoms appears, symmetrical to the cavity frequency. These lines may be attributed to cavity-induced decay under bichromatic driving.

The observed resonances in the count rate show peculiar properties. First, the resonance frequencies depend on the intensities of both injected fields. This is a clear indication that we are observing bichromatic dynamic effects and none of 
the fields may be regarded as a weak probe field. In addition, there is a dependence on the fixed frequency of the second field.

The experimentally determined position of these resonances in the lower level count rate may be summarized in a surprisingly simple condition: maxima occur, when the intracavity Rabi frequencies of the two injected fields are equal except for a constant factor of the order of unity. Denoting the amplitudes of the injected fields by $E_{1}$ and $E_{2}$ and taking into account the Lorentzian line shape of the cavity mode, this condition reads

$$
\left|\frac{2 g E_{1}}{\kappa+\mathrm{i}\left(\omega_{\mathrm{c}}-\omega_{1}\right)}\right|=\left|\frac{2 g E_{2}}{\kappa+\mathrm{i}\left(\omega_{\mathrm{c}}-\omega_{2}\right)}\right| \times f
$$

with $f$ being a constant close to one. Particularly remarkable is the width of the resonances. In the monocliromatic case, the width of all spectral structures was given either by the cavity linewidth or, for nonadiabatic effects, by the rise-time of the cavity field. In the bichromatic case, however, we found an entirely different dependence. Ilere, the width of the resonances is inversely proportional to the slope of the cavity response spectrum and therefore depends essentially on the position of the peaks relative to the cavity center. The closer to the cavity resonance the double peaks appear, the narrowcr they get. At the steepest slope of the cavity mode, which is close to the half maximum point, we have measured a width of $1 \mathrm{kIIz}$. This value is smaller than any other characteristic frequency of the system. Cavity width and transit time broadening are roughly a factor of ten larger. Inhomogeneous broadening of the atomic line does not affect the width of the resonance, as can be seen from condition (14), which is independent of the transition frequency. If we change parameters, shifting the resonance further away from the cavity center, the width increases inversely proportional to the slope of the cavity resonance line.

It should be mentioned that for certain parameters, a second pair of peaks is observed closer to the cavity center and, in accordance with the remarks above, much narrower than the first pair.

We have been able to explain the essential experimental results in the frame of a theoretical model based on the so-called Floquet states of the atom in a bichromatic field [30].

\section{Floquet analysis}

We start from a Hamiltonian analogous to Eq. (6), but now containing two fields oscillating at frequencies $\omega_{1}$ and $\omega_{2}$ with arbitrary amplitudes $E_{1}$ and $E_{2}$, respectively. To describe both fields symmetrically, we transform to a frame rotating at the average frequency

$$
\omega_{l}=\frac{1}{2}\left(\omega_{1}+\omega_{2}\right)
$$

In this frame, the HIamiltonian for bichromatic driving is

$$
\begin{aligned}
& H^{\prime}=H_{a}+\hbar \delta_{l} a^{\dagger} a+\hbar g\left(S^{+} a+S^{-} a^{\dagger}\right), \\
& H_{a}=\hbar \Delta_{l} S^{z}+\frac{\hbar}{2} \mathrm{e}^{-\mathrm{i} \nu t}\left(S^{+} \Omega_{2}+S^{-} \Omega_{1}^{*}\right)+\frac{\hbar}{2} \mathrm{e}^{\mathrm{i} \nu t}\left(S^{+} \Omega_{1}+S^{-} \Omega_{2}^{*}\right),
\end{aligned}
$$


where the delunings $\Delta_{l}, \delta_{l}$, and the frequency difference $\nu$ are defined by

$$
\Delta_{l}=\omega_{0}-\omega_{l}, \quad \delta_{l}=\omega_{\mathrm{c}}-\omega_{l}, \quad \nu=\frac{1}{2}\left(\omega_{2}-\omega_{1}\right) .
$$

The Rabi frequencies $\Omega_{1}$ and $\Omega_{2}$ of the classical driving fields are given by

$$
\Omega_{1,2}=\left|\frac{2 g E_{1,2}}{\kappa+\mathrm{i}\left(\delta_{l} \pm \nu\right)}\right| \text {. }
$$

The atomic part $H_{a}$ of Hamiltonian (17) has a periodic time dependence. It may be diagonalized by making use of Floquet's theorem, which states that the eigenfunctions $|\Psi(t)\rangle$ defined by

$$
\text { i } h \frac{\partial}{\partial t}|\Psi(t)\rangle=H_{a}(t)|\Psi(t)\rangle
$$

can be expanded in a series of harmonics of the frequency difference $\nu$ :

$$
|\Psi(t)\rangle=\mathrm{e}^{-\mathrm{i} E t} \sum_{n=-\infty}^{\infty}\left(\alpha_{1 n}|1\rangle+\alpha_{2 n}|2\rangle\right) \mathrm{e}^{-\mathrm{i} n \nu t}
$$

Using expansion (21), Eq. (20) can be solved by continued fraction methods. The smallest positive eigenvalue $E$, which will be denoted $\varepsilon$, is found in the interval $0 \leq \varepsilon<\nu / 2$. From Eq. (21) and symmetry arguments it follows that the complete spectrum of quasi-energy eigenvalues of Eq. (20) is obtained as:

$$
E= \pm \varepsilon+n \nu, \quad n=0, \pm 1, \pm 2, \ldots
$$

A section of the eigenvalue spectrum obtained in this way is shown in Fig. 9a as a function of the detuning of the first field. The slope of the levels is mainly determined by the varying intracavity intensity of $\omega_{1}$ as it is swept across the cavity resonance. In addition, there are avoided crossings of the Floquet levels if the bare atomic states involved couple. Otherwise the levels cross.

The complicated level structure has a pictorial interpretation in the atomic Bloch vector model. Each Floquet level corresponds to a Fourier component of the Bloch vector motion. Obviously, the Bloch vector does not undergo a simple Rabi nutation as in the monochromatic case. Instead, for two driving fields, many oscillation frequencies contribute to the dynamics of the Bloch vector. They are separated by half the difference $\nu$ of the two frequency components of the bichromatic field. This leads to a very complex motion of the Bloch vector.

The bichromatic effects we have observed can be explained conveniently in terms of cavity-induced transitions between the Floquet states. What distinguishes our experiment from previous studies on fluorescence of atoms under bichromatic driving is that the atoms interact with the field in an electromagnetic environment which has a strongly frequency dependent mode density. By analogy with Sec. 3, the corresponding finite response time $\kappa$ of the cavity leads to decay rates which are critically dependent on the dynamics induced by the bichromatic field.

For interpreting our experimental spectra it is important to note the double role of the driving fields. On the one hand, they essentially determine the dynamics and hence the energy splitting between the Floquet states. If none of the Floquet transitions has a frequency sustained by the cavity mode, spontaneous emission is inhibited by the same mechanism as in the monochromatic case. This is illustrated by the observation that for most experimental parameters the lower level count 


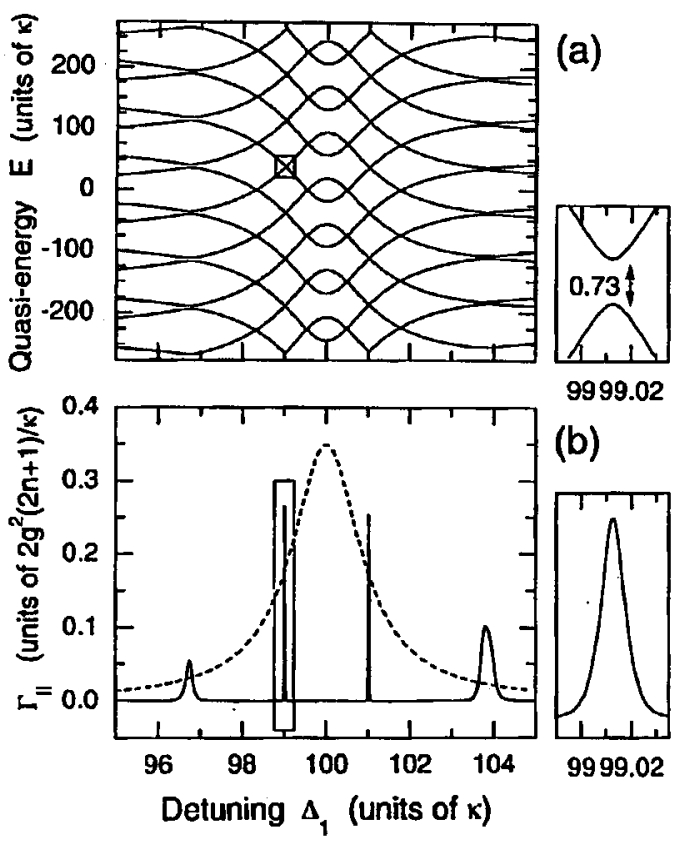

Fig. 9. (a) Floquet quasi-energy spectrum of a two-level atom in a cavity driven by a strong bichromatic field. The detuning $\Delta_{1}=\omega_{0}-\omega_{1}$ is swept, while $\Delta_{2}=\omega_{0}-\omega_{2}$, as well as the injected intensities remain fixed: $\Delta_{2}=250 \kappa ; 2 g E_{1} / \kappa=400 \kappa ; 2 g E_{2} / \kappa=4600 \kappa$. The cavity resonance is at $\Delta_{1}=100 \kappa$. (b) Decay rate $\Gamma_{\|}$of the inversion between Floquet states with energies $+\varepsilon$ and $-\varepsilon$. At the right margin, an enlarged view of a narrow avoided crossing is shown, coinciding with a peak in the decay rate.

rate is practically zero, i.e., decay is suppressed (Fig. 8). On the other hand, if a Floquet transition is resonant with the cavity, enhanced spontaneous emission should be expected. By this simple argument, the enhanced emission zone should have a width equal to the cavity linewidth $\kappa$. Experimentally, however, we found peaks in the decay rate much narrower than $\kappa$.

The apparent contradiction is resolved by taking into account that the transition rate not only depends on the density of states of the field mode, but also on the coupling between different Floquet states. As only the upper and lower bare atomic levels have a finite dipole coupling, it is important to determine the contribution of these bare levels to a given Floquet state. In other words, one has to consider the eigenvector components $\alpha_{1 n}, \alpha_{2 n}$ of a Floquet eigenvalue, defined by Eq. (21), to establish the connection between bare and dressed states. For upper state injection of the atoms, transitions between Floquet states are only possible if the initial state has a large upper level contribution while the final Floquet state must have a dominant lower level character. In the monochromatic case the composition of the dressed levels in terms of bare states is a slowly varying function of the detuning $\Delta$, essentially determined by the expression $\cos \theta$ in Eq. (11). For bichromatic driving, the situation is very different. Here, the eigenvector $\alpha$, and 
hence the transition probability, can change rapidly with detuning, if the system is close to an avoided crossing. In this way the driving fields not only determine the Floquet eigenvalues, but, through the eigenvector components, also the coupling between Floquet states.

These qualitative considerations are put on a rigorous theoretical basis in Ref. [30]. A second order Born approximation is employed to adiabatically eliminate the cavity field from the master equation. By analogy with the monochromatic case, this leads to non-Markovian decay rates for the Floquet states. The main difference is, that for bichromatic driving one has to consider transitions between all pairs of initial and final Floquet components, distinguished by the number of driving field photons involved, and sum over the respective decay rates. As a result one obtains the overall decay rate $I_{\|}^{\prime}$ for the population inversion between the Floquet states associated with $+\varepsilon$ and those with $-\varepsilon$ :

$$
\begin{aligned}
\Gamma_{\|}= & 2 g^{2}(1+2 \bar{n}) \operatorname{Re} \sum_{n, m, p, q}\left(\frac{\alpha_{2 n-} \alpha_{1 m+}^{*} \alpha_{2 p-}^{*} \alpha_{1 q+}}{\kappa+\mathrm{i} \delta_{l}-2 \mathrm{i} \varepsilon+\mathrm{i} \nu(p-q)}\right. \\
& \left.+\frac{\alpha_{2 n+} \alpha_{1 m-}^{*} \alpha_{2 p+}^{*} \alpha_{1 q-}}{\kappa+\mathrm{i} \delta_{l}+2 \mathrm{i} \varepsilon+\mathrm{i} \nu(p-q)}\right) \delta_{n-m, p-q} .
\end{aligned}
$$

This quantity was evaluated numerically and plotted in Fig. $9 \mathrm{~b}$. The correspondence with the experimental spectra of the lower level count rate is remarkable. Both feature pairs of peaks symmetrical with respect to the cavity resonance. $A$ comparison with Fig. 9a shows that the peaks appeạr precisely at those detunings, where the Floquet levels undergo an avoided crossing ( $\mathrm{cf}$. enlarged section in Fig. 9). Only in these regions, the Floquet levels with a transition frequency supported by the cavity mode couple strongly enough for enhanced emission to become possible. The fact that spontaneous decay is essentially determined by the structure and occupation of the Floquet levels and not only by the cavity mode, distinguishes this experiment from the monochromatic modification of spontaneous emission and from the static case.

The width of the peaks, too, has a natural explanation in this model. As transitions only occur close to an avoided crossing, the width of the resonances is limited by the size of the avoided crossing region. This width is inversely proportional to the slope of the unperturbed Floquet levels at the avoided crossing as a function of $\omega_{1}$, which, on the other hand, follows the cavity line shape. Therefore, the peaks are narrower, if they appear at frequencies $\omega_{1}$ close to the cavity center, where the slope of the mode density is steeper. Again, this is in perfect agreement with experimental observations.

Thus we think the interpretation of our results as cavity-modified transitions between Floquet states of a two-level atom provides a consistent explanation of the major features observed in the experiment.

Open questions, like the detailed dependence of the position of the peaks on the experimental parameters must be solved by a careful analysis of the transient behavior of the Bloch vector motion as the atoms traverse the cavity. This can be done by integrating the Bloch equations obtained from the bicliromatic master equation. 


\section{Absence of absorption}

In the experiments described so far, atoms were always injected in the upper level of the Rydberg transition. In this way we probed the dynamic modification of the combined rate of spontaneous emission and thermally induced transitions in the cavity. Our experimental setup allows us, alternatively, to send the atoms into the cavity in the lower state. This is achieved by first laser-exciting the intermediate level $52 P$ and subsequently populating $53 S$, the lower level of the Rydberg transition, in a microwave excitation zone in front of the cavity entrance. Thus we could test if the rate of absorption of thermal plotons was subject to the same dynamic modification as was found for emission.

We performed the experiment at a temperature of $4.2 \mathrm{~K}$, corresponding to an average number of $\bar{n}=2.9$ thermal photons in the resonant cavity mode. In this regime, we did not expect a substantial difference between upper and lower level injection. Thermally induced transitions and spontancous emission on the one hand and absorption of thermal photons on the other hand should both lead to the same thermal distribution of population among the two levels, the transit time being large enough to achieve thermalization.

The surprising experimental result, however, was that the expected absorption of thermal photons in the cavity was completely absent, even when we tuned the driving fields precisely where we had observed Floquet resonances in the upper level injection scheme (Sec. 4). A lower level injection spectrum equivalent to Fig. 8 displayed no resonances at all. Instead, suppression of thermal transitions was observed over the full parameter range.

As a further test of this apparent asymmetry between emission and absorption, we examined the intermediate case with occupation in both upper and lower level.

We realized this situation experimentally by tuning the second (constant) microwave field such that the upper level was pumped by thermal photons. We achieved an upper to lower level occupation ratio of two over three.

When we added and scanned the first microwave field, we typically obtained spectra like the one presented in Fig. 10. The observations are in correspondence with our earlier results: when the first field is tuned to a Floquet resonance, atoms in the upper level are subject to enhanced transitions to the lower level, while the atoms originally in the lower level are not affected. Consequently, at resonance the upper level occupation is largely decreased, while the lower level occupation rises, i.e., the less populated level is emptied into the more populated one.

This is only possible, if the transition rate from the upper to the lower level is significantly larger than the rate in the opposite direction, i.e.,

$$
\Gamma_{1 \rightarrow 2} \gg \Gamma_{2 \rightarrow 1} \text {. }
$$

This asymmetry between transition rates is reminiscent of some schemes for maser action in the absence of inversion. Part of the current theoretical work on the problem is devoted to the question, if destructive interference of transition amplitudes between Floquet levels could be at the origin of the asymmetry.

An alternative explanation starts from the fact that due to spontaneous 


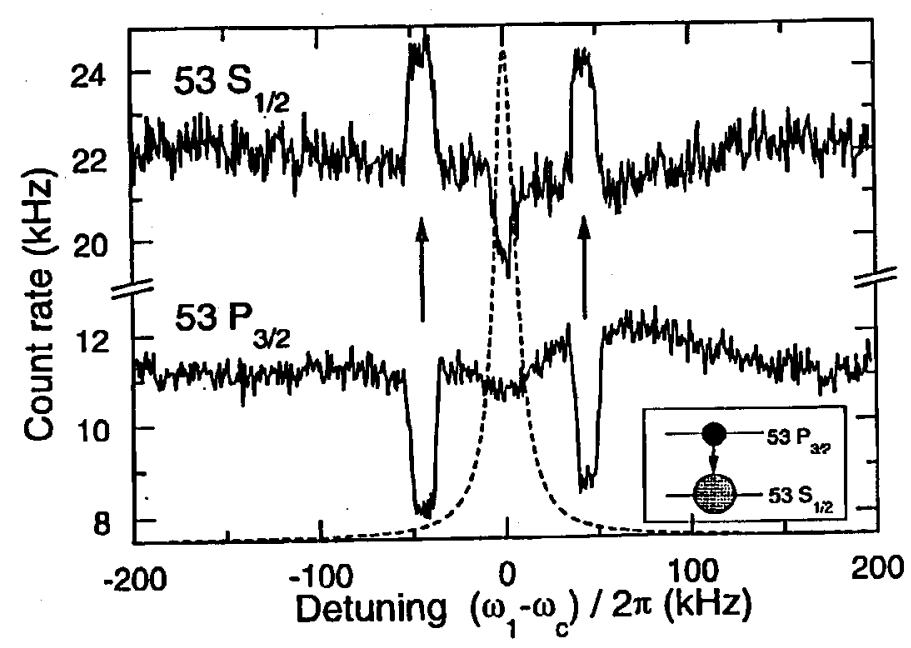

Fig. 10. Rydberg count rates for lower level injection with a thermal occupation of the upper level, in the presence of a bichromatic driving field. At the narrow resonances, the cavity induces transitions from upper to lower level only (as indicated by arrows).

emission, there is always a small imbalance in the transition rates

$$
\frac{\Gamma_{1 \rightarrow 2}}{\Gamma_{2 \rightarrow 1}}=\frac{1+\bar{n}}{\bar{n}}
$$

At a thermal photon number $\bar{n} \approx 2.9$, this ratio is close to unity, so that no large asymmetry can be expected. However, the actual number of thermal photons in the cavity decreases, when a beam of lower state atoms passes through the resonator. The reason is that thermal photons are continuously absorbed by the atoms and carried out of the cavity. This happens at a rate which is essentially the inverse atomic transit time $T^{-1}$ multiplied by the number of atoms $N$ in the cavity. On the other hand, the cavity mode tries to restore the state of thermal equilibrium, which happens at the cavity damping rate $r$. If the flux of atoms through the cavity is large enough, the steady state thermal photon number $\vec{n}$ which is determined by the two rates $N / T$ and $\kappa$, can be substantially smaller than 2.9. According to (25), in this case the atoms experience a larger asymmetry. Ilowever, we estimate that it would take at least 200 atoms in the cavity to explain the spectra we have obtained. As on the average there are only ten to thirty atoms in the cavity, the observed imbalance between the upper and lower level decay rates continues to be a theoretical challenge.

\section{Summary}

We have investigated two-level Rydberg atoms interacting with a single field mode of a moderate- $Q$ cavity in the strong external driving regime. The intensities employed in our experiments cover the range from above saturation almost up to the ionization threshold, a region not explored so far. 
Our results show that the intense driving field, together with the strong frequency dependence of the cavity mode density leads to decay terms with non-Markovian characteristics. They make cavity-induced transitions critically dependent on strength and detuning of the injected field. If the strong driving field has only a single frequency component and is tuned close to resonance, it suppresses decay in the system, i.e. practically decouples the atom from the cavity field.

More complex spectral features are found for a bichromatic driving field. In this case, cavity-induced decay is restricted to very narrow resonances, appearing at certain detunings and intensities of the external fields. They can be traced back to the fact that the bichromatic driving creates a complex energy level structure best described by Floquet states. We were able to control the transition frequencies and widths of cavity-induced transitions between these Floquet states by changing the intensities and the detunings of the driving fields. In this way we could switch on and off cavity-induced decay almost arbitrarily. Further research should yield new insights into strongly driven systems. An intriguing question is the origin of the observed asymmetry between cavity-induced emission and cavity-induced absorption in our setup.

On the theoretical side, the calculation of decay rates in the Born approximation allowed us to explain the major features of the experiment. For a full understanding of the dynamics in a strongly driven cavity, however, a numerical solution of the full master equation is desirable. In such a treatment, transient effects could be taken into account, which due to the occurrence of multiple avoided crossings in the entrance and exit phase are expected to be of considerable importance.

\section{References}

[1] E.T. Jaynes, F.W. Cummings, Proc. IEEE 51, 89 (1963).

[2] D. Meschede, I. Walther, G. Müller, Phys. Rev. Lett. 54, 551 (1985).

[3] G. Rempe, H. Walther, N. Klein, Phys. Rev. Lett. 58, 353 (1987).

[4] G. Rempe, F. Schmidt-Kaler, II. Walther, Phys. Rev. Lett. 64, 2783 (1990).

[5] M.G. Raizen, R.J. Thompson, R.J. Brecha, H.J. Kimble, H.J. Carmichael, Phys. Rev. Lett. 63, 240 (1989).

[6] R.J. Thompson, G. Rempe, II.J. Kimble, Phys. Rev. Lett. 68, 1132 (1992).

[7] F. Bernardot, P. Nussenzweig, M. Brune, J.M. Raimond, S. Haroche, Europhys. Lett. 17, 33 (1992).

[8] E.M. Purcell, Phys. Rev. 69, 681 (1946).

[9] D.J. Heinzen, J.J. Childs, J.E. Thomas, M.S. Feld, Phys. Rev. Lett. 58, 1320 (1987).

[10] P. Goy, J.M. Raimond, M. Gross, S. Haroche, Phys. Rev. Lett. 50, 1903 (1983).

[11] R.G. Hulet, E.S. Hilfer, D. Kleppner, Phys. Rev. Lett. 55, 2137 (1985).

[12] W. Jhe, A. Anderson, E.A. Hinds, D. Meschede, L. Moi, S. Haroche, Phys. Rev. Lett. 58, 666 (1987).

[13] W. Lange, H. Walther, Phys. Rev. A 48, 4551 (1993).

[14] F. Casagrande, L.A. Lugiato, W. Lange, H. Walther, Phys. Rev. A 48, 790 (1993). 
[15] P. Alsing, II.J. Carmichael, Quantum Opt. 3, 13 (1991).

[16] S.Y. Kilin, T.B. Krinitskaya, J. Opt. Soc. Am. B 8, 2289 (1991).

[17] G.S. Agarwal, W. Lange, H. Walther, Phys. Rev. A 48, 4555 (1993).

[18] M. Lewenstein, T.W. Mossberg, R.J. Glauber, Phys. Rev. Lett. 59, 775 (1987).

[19] G.S. Agarwal, Phys. Rev. A 19, 923 (1979).

[20] Y.S. Bai, A.G. Yodh, T.W. Mossberg, Phys. Rev. Lelt. 55, 1277 (1985).

[21] C. Cohen-Tannoudji, J. Dupont-Ruc, G. Grynberg, Atom-Photon Interactions Basic Processes and Applications, Wiley, New York 1992, Ch. VI.

[22] B.R. Mollow, Phys. Rev. 188, 1969 (1969).

[23] Y. Zhu, Q. Wu, A. Lezama, D.J. Gauthier, T.W. Mossberg, Phys. Rev. A 41, 6574 (1990).

[24] H.S. Freedhoff, Z. Chen, Phys. Rev. A 41, 6013 (1990).

[25] G.S. Agarwal, Y. Zhu, D.J. Gauthier, T.W. Mossberg, J. Opt. Soc. Am. B 8, 1163 (1991).

[26] Z. Ficek, H.S. Freedhoff, Phys. Rev. A 48, 3092 (1993).

[27] G. Floquet, Ann. de l'Ecole Norm. Sup. 12, 47 (1983).

[28] B.R. Mollow, Phys. Rev. A 12, 1919 (1975).

[29] E. Courtens, A. Szöke, Phys. Rev. A 15, 1588 (1977).

[30] G.S. Agarwal, W. Lange, H. Walther, unpublished. 